\title{
Laterality Effect for Faces in Chimpanzees (Pan troglodytes)
}

\author{
Christoph D. Dahl, ${ }^{1,2}$ Malte J. Rasch, ${ }^{3}$ Masaki Tomonaga, ${ }^{1}$ and Ikuma Adachi ${ }^{4}$ \\ ${ }^{1}$ Primate Research Institute, Kyoto University, Section Language and Intelligence, Inuyama, Aichi 484-8506, Japan, ${ }^{2}$ Department of Psychology, National \\ Taiwan University, Taipei 106, Taiwan, ${ }^{3}$ State Key Laboratory of Cognitive Neuroscience and Learning, Beijing Normal University, 100875 Beijing, China, \\ and 4 Primate Research Institute, Kyoto University, Center for International Collaboration and Advanced Studies in Primatology, Aichi 484-8506, Japan
}

Face perception in humans is governed more by right-hemispheric than left-hemispheric neural correlate. Some but not all neurophysiological studies depict a right-side dominance for face responsive neurons in the brains of macaques. Hence, it is an open question whether and to what extent a right-hemisphere preference of processing faces exists across primate brains. We investigated chimpanzees discriminating chimeric faces of chimpanzees and humans, i.e., the combination of either left or right sides of a face vertically flipped and merged into a whole face. We found an effect of choosing the left-chimeric face more often than the right-chimeric face as being the one of the two that is closer to the original face, reflecting an advantage for the right side of the brain to process faces, as reported in humans. Moreover, we found a modulation by age of the participants, suggesting that the exposure history with a particular category shapes the right-hemispheric neural correlate to a configural/holistic processing strategy. In other words, the findings in chimpanzee participants parallel those in human participants and are suggestive for similar neural machineries in the occipital-temporal cortices in both species.

\section{Introduction}

In humans, the right hemisphere of the brain is generally more reliable in discriminating faces (Geffen et al., 1971; Rizzolatti et al., 1971), which becomes evident in a quicker (Broman, 1978) and more accurate (Hilliard, 1973; Ellis and Shepherd, 1975) identification of faces presented to the left than to the right visual field. Studies with lateral brain-damaged patients showed selective functional impairments in face processing (Levy et al., 1972; Sergent and Signoret, 1992; Bouvier and Engel, 2006); neuroimaging studies showed stronger activation in the right than in the left hemisphere (Gauthier et al., 1999; Ishai et al., 2000; GrillSpector et al., 2004; Golarai et al., 2007) or only activation in the right and none in the left hemisphere (Campanella et al., 2001; Morris et al., 2007; Rotshtein et al., 2007).

In nonhuman primates, evidence for lateralization in face perception is mixed. Overman and Doty (1982) showed so-called chimeric faces (i.e., a face where one side is vertically flipped to the other side) to humans and macaques and found an advantage

Received Feb. 7, 2013; revised July 2, 2013; accepted July 4, 2013.

Authors contributions: C.D.D. and I.A. designed research; C.D.D. and I.A. performed research; C.D.D., M.J.R., M.T. and I.A. contributed reagents/materials/analysis tools; C.D.D. and M.J.R. analyzed data; C.D.D. wrote the paper.

This work was supported by the Ministry of Education, Science, Sports and Culture, Japan [Grant 60111986 for Scientific Research on Innovative Areas, "Face Perception and Recognition"; Specially Promoted Research 24000001; Grant (S) 70237139 for Scientific Research; and Grant (B) 22700270 for Young Scientists (I.A.)], the Japan Society for the Promotion of Science (C.D.D.), and the German Academic Exchange Service (M.J.R.). We thank the staff of the Language and Intelligence Section for their help and useful comments. We also thank the Great Ape Research Institute and the Kumamoto Chimpanzee Sanctuary for kindly providing photographs of their animals, and the Center for Human Evolution Modeling Research at the Primate Research Institute, for daily care of the chimpanzees. The authors declare no competing financial interests.

Correspondence should be addressed to one of the following: Christoph D. Dahl, Primate Research Institute, Kyoto University, Section Language and Intelligence, Inuyama, Aichi 484-8506, Japan. E-mail: dahl@pri.kyotou.ac.jp, christoph.d.dah@@gmail.com; or Ikuma Adachi, Primate Research Institute, Kyoto University, Center for International Collaboration and Advanced Studies in Primatology, Inuyama, Aichi 484-8506, Japan. E-mail: adachi@pri.kyoto-u.ac.jp.

DOI:10.1523/JNEUROSCI.0590-13.2013

Copyright $\odot 2013$ the authors $\quad 0270-6474 / 13 / 3313344-06 \$ 15.00 / 0$ of processing for the left-chimeric faces in humans, but not in macaques. However, a split-brain study in monkeys showed a clear left visual field advantage (Hamilton and Vermeire, 1988), apart from a series of unsuccessful attempts (Hamilton, 1977, 1983). Indistinct are the activity profiles in the brains of macaques: while there is convincing evidence of hemispheric asymmetries for faces in favor of the right hemisphere using a dual-activity mapping technique (Zangenehpour and Chaudhuri, 2005) based on the induction of the immediate early gene zif268 (Chaudhuri et al., 1997), there are disillusioning insights from functional magnetic resonance imaging (fMRI) in awake and anesthetized macaques showing no lateralized response characteristics (Bell et al., 2011; Popivanov et al., 2012) or lateralized response characteristics, but not predominantly in the right hemisphere ( $\mathrm{Ku}$ et al., 2011). In chimpanzees, chimeric faces of happy and neutral expressions were easier to discriminate when the happy half fell into the left visual field (Morris and Hopkins, 1993). However, a left visual field advantage did not occur in a match-to-sample paradigm (Plotnik et al., 2003).

Given this inconsistency, further clarification into the extent to which there is hemispheric specialization for face perceptionand more specifically face discrimination-in nonhuman primates is needed. We address the question: do chimpanzees exhibit a right-hemispheric advantage for the discrimination of faces? Given the similar design principle of the chimpanzee and human visual systems, we predicted that chimpanzees, like humans (Gilbert and Bakan, 1973), would perceive a chimeric face containing the left halves of a face as being more similar to the original face than a face containing the right halves of that face.

\section{Materials and Methods}

Participants. Four chimpanzees (Pan troglodytes; 1 male juvenile, 1 female juvenile, both $\sim 10$ years old; 2 female adults, both $\sim 30$ years old) participated in this study. At the Primate Research Institute, chimpanzees live in groups of 14 individuals and have access to environmentally 
enriched outdoor $\left(770 \mathrm{~m}^{2}\right)$ as well as indoor compounds. Over the years, they participated in various types of computer-controlled perceptualcognitive experiments. In addition, 12 human subjects (average age, 28.9 years; SD [standard deviation], 6.1 years) participated as control subjects to compensate for the low number of chimpanzee participants. All experiments were performed in accordance with the 2002 version of the Guidelines for the Care and Use of Laboratory Primates by the Primate Research Institute, Kyoto University. The experimental protocol was approved by the Animal Welfare and Care Committee of the same institute.

Stimuli. We used black-and-white pictures of eight chimpanzee as well as eight human individuals. The faces were normalized for luminance and contrast, placed in an image canvas of $533 \times 702$ pixels and presented at $40 \mathrm{~cm}$ distance, corresponding to $\sim 10.7 \times 14.25^{\circ}$ of visual angle. In addition to the original picture, for each individual the two chimeric faces (left-chimeric and right-chimeric faces) were generated. The two sides (left and right) for the chimeric faces were extracted by cutting the original face in the vertical dimension. The cutting line was placed through the tip of the nose and the mid-intereye distance. Due to the constraint of a most accurate separation of the left and right sides, we used a limited set of eight original faces per class (chimpanzee and human faces). In addition to the eight chimeric faces, we used an additional set of 12 faces per class with two pictures of each individual. These stimuli were used for the control trials (see below, Procedure).

Apparatus. Chimpanzees participated in pairs (mother and daughter/ son). Stimuli were presented at a 17 -inch liquid-crystal touch-panel display $(1280 \times 1024$ pixels $)$ controlled by custom-written software under Visual Basic 2010 (Microsoft). Chimpanzees sat in two connected experimental chambers (each $\sim 2.5 \mathrm{~m}$ wide, $2.5 \mathrm{~m}$ deep, $2.1 \mathrm{~m}$ high), while the experimenter sat outside the booth separated by transparent acrylic panels. The display was mounted into the acrylic panel. We tried to keep the distance between the display and the participant constant at $\sim 40 \mathrm{~cm}$. One degree of gaze angle corresponded to $\sim 0.7 \mathrm{~cm}$ on the screen at a 40 $\mathrm{cm}$ viewing distance. The participants responded by touching the display surface with a finger. The display was protected from deterioration by a transparent acrylic panel fitted with an armhole $(10 \times 47 \mathrm{~cm})$ allowing hand contact with the display. Below the display, a food tray was installed in which pieces of food reward was delivered by a custom-designed feeder. Display and feeder were automatically controlled by the same program that controlled the display of the stimuli. Human participants were tested in a dedicated setup for psychophysical experiments under conditions comparable to the testing of chimpanzees. We did not use a chin rest, but instructed the participants to focus on the midpoint of the screen upon cue stimulus presentation and to keep their head position constant during the experiment. The degree of gaze angle was comparable to that of the chimpanzee participants.

Procedure. We used a delayed matching-to-sample paradigm (Fig. 1A, caption). The procedure was equivalent for chimpanzee and human participants. Each test trial was initiated by the participant by pressing a centrally presented button on the screen. This ensured that participants were initially looking at the midpoint of the cue stimulus. Test trials consisted of an original face picture (cue) centrally presented for $750 \mathrm{~ms}$, followed by an intertrial interval of $500 \mathrm{~ms}$, followed by the two chimeric faces of both left and right sides of the previously presented original face. A decision for either of the two chimeric faces was required, indicated by touching the stimulus. The location of the two chimeric faces was randomized and horizontally arranged with a spatial separation of $20 \mathrm{~mm}$. Since for these trials, there is no correct or incorrect answer, we rewarded the chimpanzee participant regardless of behavior. To avoid random or idiosyncratic response strategies, we interspersed these test trials among no-test trials that contained a cue ( 1 individual) followed by a match (the same individual, but a different picture) and a distractor (different individual) at a ratio of 1:5. We divided the sequence into runs of 50 trials and alternated between runs of chimpanzee and human stimulus presentations. This task was restricted to chimpanzees that exceeded a baseline of correct discrimination of $85 \%$ in two consecutive sessions of unaltered chimpanzee and human faces (similar to the no-test trials). These results were reported previously (Dahl et al., 2013). Two of six participants from that study ( 1 female juvenile, 1 female adult) were not qualified to proceed with the chimeric face experiment. For the others, we ran 300 chi- meric trials per stimulus class. These runs were recorded in $12.5 \pm 1.9 \mathrm{~d}$. The human participants did two runs of 24 trials, repeating each face three times. The order of runs and stimuli within the runs were fully counter-balanced across and within participants.

Data analysis. The dependent variable was (1) the cumulative number of decisions for the left-chimeric and the right-chimeric face and (2) the proportion of choices favoring the left-chimeric face over the rightchimeric face. We used binomial and Pearson's correlation tests to evaluate lateralization. Due to the low number of participants, we ran randomization procedures drawing values from independent Gaussian distributions with mean and SD of the original datasets and correlated these values. The $95 \%$ confidence intervals (CIs) were determined based on 1000 repetitions of this procedure.

\section{Results}

We tested the response biases of chimpanzee participants for chimeric faces of chimpanzees and humans containing either left or right face sides ( 1 side vertically flipped; Fig. $1 A$ ). An unaltered cue stimulus (e.g., original Face 1) was centrally presented followed by the left and right side versions of the same face (Face 11, Face 1r). We found a systematic decision bias toward the leftchimeric faces of chimpanzees in all of the four chimpanzee participants, which was significant after 126 trials ("normal" trials not included) (binomial test: $N=126, p<0.05$; Fig. $1 B$, left, $E$, left, arrowheads). Three of four chimpanzees showed a significant bias for left-chimeric faces of humans, which was significant at individual level after maximally 85 trials $(N=85, p<0.05$; Fig. $1 B$, right, $E$, right, black arrowheads). Using an iterative randomization procedure (1000 repetitions), we generated binary sequences $(1,-1)$, representing decisions for left-chimeric and right-chimeric faces, by cumulatively adding up the number in the individual sequences. We finally computed the 95\% CIs from the randomized dataset and compared the actual values with these. We showed that all of the participants were with 5\% confidence beyond the criteria of a random left-right chimeric face bias for chimpanzee faces, while for human faces three of the participants were beyond the criteria and one almost reached the level of $95 \%$ CI. The $95 \%$ CI is indicated by the light red color patches along the zero line on the $x$-axis (Fig. $1 B$ ). More critically, the left-chimeric face bias was modulated by the species of the face stimulus, i.e., greater for old chimpanzees in human than in chimpanzee faces and greater for young chimpanzees in chimpanzee than in human faces. Among the four participants, we found a negative correlation between left-chimeric face bias for chimpanzee and human faces being significant at the $5 \%$ level after 208 trials ("normal" trials excluded; $r_{(2)}=-0.90, p<0.05$; Fig. $1 G)$. We tested the hypothesis that the correlation among the two datasets arose by chance by drawing values from two independent distributions. We extracted four values for each of the two datasets (left-chimeric face bias for chimpanzee and human stimuli) from respective Gaussian distributions with mean and $\mathrm{SD}$ of the original datasets and correlated these values (see Materials and Methods). We found that a random effect can be excluded at the 5\% significance level. More interestingly, in our recent study, we showed a systematic modulation of discrimination performance by age (Dahl et al., 2013): young chimpanzees $(\sim 10$ years $)$ showed better discrimination abilities for chimpanzee than human faces, while old chimpanzees ( $\sim 30$ years) showed better discrimination abilities for human than chimpanzee faces. We here reconsider this dataset and found that the laterality bias in each participant after 300 trials (left-chimeric face bias of chimpanzee and human faces) is closely linked to the classification performance index for both chimpanzee and human stimulus sets (Dahl et al., 2013; performance index: scores 

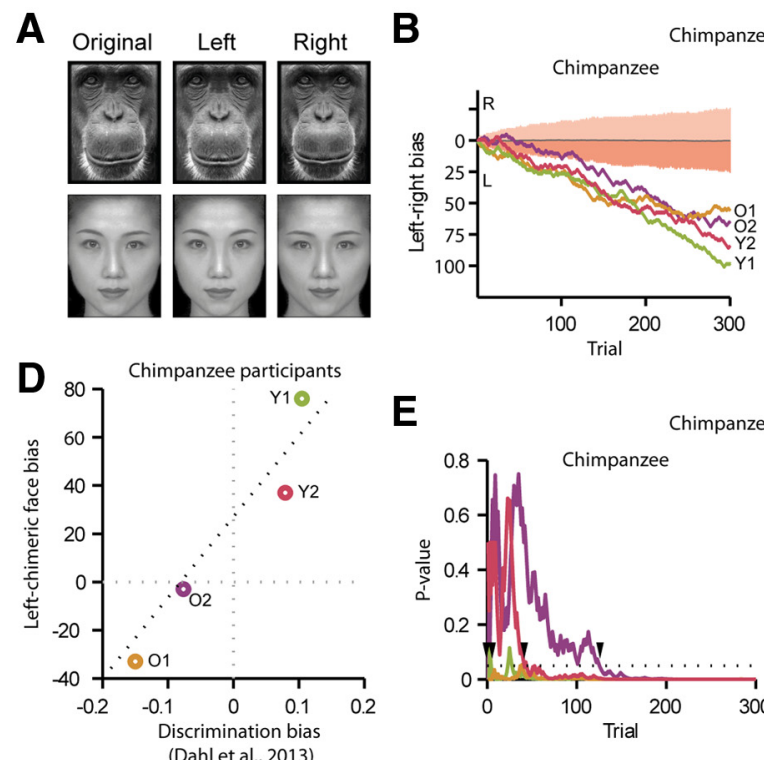

E
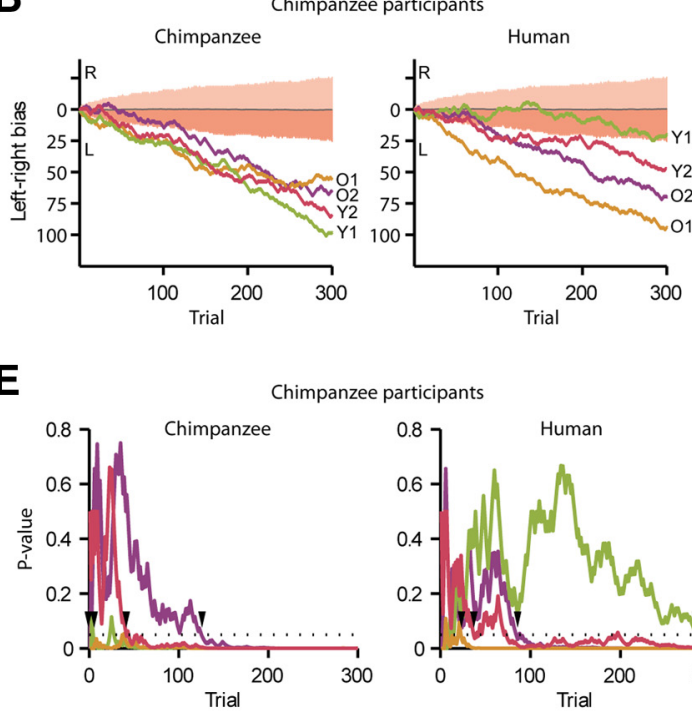

C

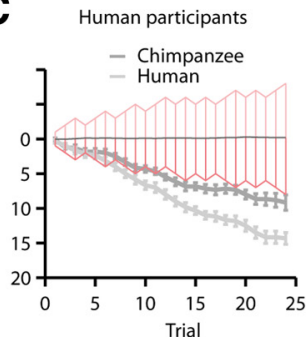

$\mathbf{F}$
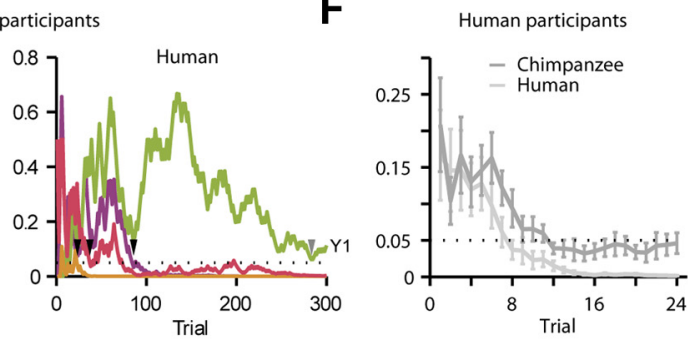

G

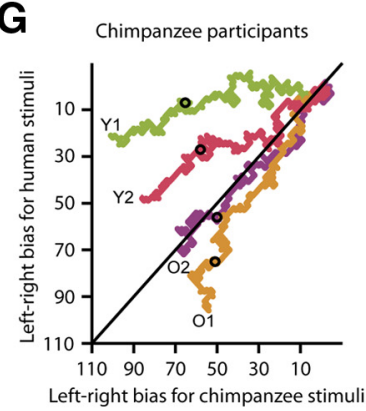

H

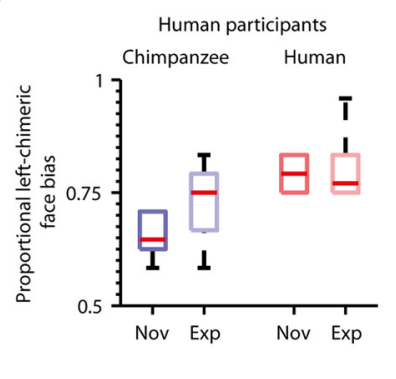

Figure 1. Laterality effect. $A$, Example stimuli of chimpanzee and human original and chimeric faces. B, Left-chimeric face bias for chimpanzee participants. Colored lines indicate the participants' responses plotted as a cumulative function of left-right decisions ( $x$-axis) over time $(y$-axis, trials). The light red bar distribution around the zero line shows an iterative randomization procedure of left-right decisions indicating the significance level of 5\%. C, Left-chimeric face bias for human participants. Gray lines indicate the participants' responses plotted as a cumulative function of left-right decisions ( $x$-axis) over time $(y$-axis, trials) for chimpanzee (dark) and human (light) faces. $\boldsymbol{D}$, Correlation of laterality biases and classification performance indices of chimpanzee participants. The $x$-axis shows the discrimination bias, i.e., the subtraction of performance scores for chimpanzee faces from those for human faces. Positive values indicate better performance for chimpanzee than human faces. The $y$-axis indicates the left chimeric face bias, i.e., the subtraction of the left-chimeric face biases for chimpanzee faces from those for human faces. Positive values indicate a stronger bias for chimpanzee than human faces. Individual participants are marked by a colored circle. $\boldsymbol{E}$, Distributions of $p$ values for chimpanzee participants. Binominal tests were conducted for each point in time to determine the effect of left-chimeric face biases. The color lines represent the participants, the dashed line represents the significance level of $5 \%$, while the arrowheads mark the individual significance level of $5 \%$ (black) and $6.5 \%$ (gray, $\mathrm{Y} 1$ for human faces). $\boldsymbol{F}$, Distributions of $p$ values for human participants. Binomial tests were conducted similar to chimpanzee participants. G, Negative correlation between the left-chimeric face biases for chimpanzee and human face stimuli. Colored lines indicate the participants; the gray-yellow background indicates the level of significance (while black circles mark $p<0.05)$. $\boldsymbol{H}$, Level of expertise. The performances of chimpanzee-novice and chimpanzee-expert human participants were contrasted for chimpanzee and human chimeric faces.

for chimpanzee-human faces; $r_{(2)}=0.97, p<0.05$; Fig. $1 D$ ) using a Pearson's correlation test. To test the hypothesis that the correlation among the two datasets arose by chance by drawing values from two independent distributions, we extracted four values for each of the two datasets (left-chimeric face bias for chimpanzee and human stimuli and the discrimination experiment of both chimpanzee and human faces; Dahl et al., 2013) from respective Gaussian distributions with mean and SD of the original datasets and correlated these values. A random effect can be excluded at the $5 \%$ significance level.

To account for the low number of chimpanzee participants, we tested 12 human participants under the analogous hypothesis. We found a systematic decision bias toward the left-chimeric faces of humans in all human participants (binomial test: $N=24$, $p<0.05$; Fig. 1C,F, light gray line). Eleven of 12 humans showed a significant bias for left-chimeric faces of chimpanzees $(N=24$, $p<0.05$; Fig. 1C,F, dark gray line). As for the chimpanzee participants, we generated binary sequences $(1,-1)$, representing decisions for left-chimeric and right-chimeric faces, by cumulatively adding up the number in the individual sequences. We showed that at the population level humans were with $5 \%$ confidence beyond the criteria of a random left-right chimeric face bias for chimpanzee and human faces. The $95 \% \mathrm{CI}$ is indicated by the light red color patches along the zero line on the $x$-axis (Fig. $1 C)$. In addition, we tested whether the level of expertise with chimpanzee faces causes a difference in the number of decisions toward the left-chimeric face. We compared chimpanzee-naive participants $(N=6)$ with students $(N=6)$ from the Primate Research Institute of Kyoto University, who were exposed to 13 chimpanzees at a regular basis over a maximum of 5 years. Using a mixed-model ANOVA (with stimulus class and expertise level as fixed factors), we could not find a significant interaction between the factors stimulus class and expertise level $(p>0.17)$. However, using a two-sample $t$ test, we found a difference in the proportions of choosing left-chimeric chimpanzee above leftchimeric human faces (proportion choosing left-chimeric hu- 
man faces minus proportion choosing left-chimeric chimpanzee faces) between the two groups $\left(t_{(10)}=-1.93, p<0.05\right.$; mean "novices," 0.05; mean "experts," 0.16; 1-tailed; Fig. $1 H$ ), suggesting a stronger lateralized processing of chimpanzee faces with as little as a few years of exposure to a limited number of chimpanzees (expert group) as opposed to the novice participants with no specific exposure history to chimpanzees. Given the relatively weak effect size, the exact nature of modulation by expertise needs to be further established.

In summary, we found a left-chimeric face bias in chimpanzees for both chimpanzee and human faces, as reflected in the number of choices favoring left-chimeric faces over rightchimeric faces. Chimpanzees exhibit a right-hemispheric advantage for the processing of faces. In context with a recent study (Dahl et al., 2013), the current results reflect the differential contributions of early and late processes in face perception that drive the perceptual system to chimpanzee faces early in life (perceptual narrowing; Sugita, 2008) and shape it in the long term along the critical dimensions of the faces exposed to (perceptual learning; Goldstone, 1998). In detail, the effect size was modulated by the individual discrimination ability in both types of faces, revealed by a positive correlation between the performance in chimpanzee and human faces (Dahl et al., 2013) and the righthemispheric dominance of processing faces in the brain: the young chimpanzees showed a stronger left-chimeric face bias in chimpanzee than human faces along with a better performance in discriminating chimpanzee than human faces. The opposite trend was found for old chimpanzees, which had an immense exposure to human faces over their lives. Fully in accordance with the findings in chimpanzees, findings in human participants showed a left-chimeric face bias toward conspecific faces and a weakened effect for faces of expertise.

\section{Discussion}

While in humans insights into lateralized processing of faces in the brain exist on various levels, for example, lateralized brain activation (Kanwisher et al., 1997; McCarthy et al., 1997), splitbrain patients (Gazzaniga and Smylie, 1983; Miller et al., 2002), left-gaze bias (Mertens et al., 1993; Butler et al., 2005), left-visual field advantages (perceptual judgments of gender, age, identity, expression, likeness, and attractiveness; Gilbert and Bakan, 1973; Burt and Perrett, 1997; Butler et al., 2005), in nonhuman primates the findings are less consistent: the left-visual field advantage was tested in chimpanzees in a match-to-sample paradigm (with no temporal delay between cue and match-distractor pair; Plotnik et al., 2003), similar to the one used in the current study. The study found no bias toward the left-chimeric faces, suggesting no left-visual field advantage in chimpanzees. However, the difference between that and our study was that in their study chimpanzees were extensively trained on matching chimeric faces (including a chimeric face as cue) before the actual test condition, which included an original (unaltered) face (cue) followed by the left and right chimera. The training might lead to idiosyncratic response strategies to successfully differentiate between the two chimeric faces of one individual resulting in unbiased responses in the testing phase (where both "match" and "distractor" are correct). In contrast, the chimpanzees in our study have never experienced chimeric faces in their past. Further in support of a left-visual field advantage, chimpanzees were tested on the ability to discriminate happy from neutral human faces (Morris and Hopkins, 1993): the likelihood of selecting the smiling half was larger when it fell into the left hemispatial field. A PET study in chimpanzees showed no right hemispheric lateral- ization when comparing face and object activations. However, a general patch of activation was found in the right ventral temporal sulcus (Parr et al., 2009), which resulted from comparisons of faces or objects against a resting period. This is suggestive, but not critical enough to claim face-selective patches. Patches in the face-versus-resting condition can arise from feature dimensions similar to those of objects and not selectively from faces. Behavioral evidence in terms of a left-gaze bias toward conspecific and human faces was found in laboratory-raised macaques, while domestic dogs showed a left-gaze bias selectively to human, not conspecific, and monkey faces (Guo et al., 2009), suggesting a face-sensitive and species-sensitive gaze asymmetry. The left-gaze bias reflects to some degree both the hemispheric lateralization in face processing and the sampling of salient and relevant facial information from local facial features (Butler et al., 2005). Striking behavioral evidence for brain asymmetries in monkey comes from split-brain studies: an inversion effect for faces has been shown involving the right hemisphere (Vermeire and Hamilton, 1998), indicating that monkeys process faces configurally in the right hemisphere, but when required they can employ part-based processing in both hemispheres. Further, split-brain monkeys showed advantages in learning and remembering facial discriminations in the right hemisphere (Vermeire et al., 1998). The brain activation profiles caused by face stimulation are not entirely consistent across the literature. A study using a dual-activity mapping technique (Zangenehpour and Chaudhuri, 2005), based on the induction of the immediate early gene zif268 (Chaudhuri et al., 1997), provided direct evidence at the cellular level for a right-hemispheric asymmetry of face patches throughout the inferotemporal cortex in macaques. However, fMRI in awake and anesthetized macaques showed lateralized response characteristics, but not predominantly in the right hemisphere (Ku et al., 2011). While some studies are suggestive for a humanlike brain architecture (Tsao et al., 2008), by showing righthemispheric face patches from area $\mathrm{V} 4$ to rostral temporal cortex (Tsao et al., 2003), others show equivalently pronounced face patches between the hemispheres (Bell et al., 2011; Popivanov et al., 2012). Early work using single-cell recordings revealed clear evidence for asymmetrically right-hemispheric representations of face-selective neurons in the superior temporal sulcus of the monkey brain (Perrett et al., 1988). Together, individual findings are supporting the notion that monkeys, similar to humans, process faces dominantly in the right hemisphere of the brain. However, in a broad perspective, it is still far from clear whether human and monkey brains show similarities in the distribution of face selectivity. The factors that determine the large variability in monkey face patches across studies remain elusive to a great extent.

In the current study, we found an asymmetric contribution between the right and left sides of chimpanzee and human faces, reflected in a preference to select the left-chimeric over the rightchimeric face. An explanation for this effect is that these faces were asymmetrically presented to the two hemispheres by, for example, a skewed initial landing fixation (Hsiao and Cottrell, 2008). This, however, was unlikely since each trial was initiated by a start button that guides the participant's eye gaze to the center of the screen and, upon presentation of the cue stimulus, to the center of the face. Further, following the cue stimulus and the intertrial interval, the two (left and right) chimeric faces were presented horizontally arranged to both sides of the screen in a counter-balanced fashion. Our results not only reflect a leftchimeric face bias, but a relative contribution of the tuning of the face system toward one or the other face class to the left-chimeric 
face bias. We double-dissociate the findings using a human control group with different levels of expertise with chimpanzee faces. Hence, it seems very unlikely that our findings result from an asymmetric distribution.

More interestingly, left-lateralized processing of one class of faces (chimpanzee or human faces) correlates with the general discrimination performance (Dahl et al., 2013) for that type of face. This suggests that there is a qualitative difference in the processing of "expert" faces (e.g., conspecific and/or heavily exposed faces) as opposed to "nonexpert" faces (e.g., nonconspecific and/or less exposed faces), as previously shown in humans (Michel et al., 2006; Kelly et al., 2007; Dahl et al., 2009) and macaque monkeys (Dahl et al., 2009, 2010, 2011): the more exposure toward one type of faces, the more the perceptual system relies on face-specific right-hemispheric processing, also shown in sheep (Peirce et al., 2000). However, the perceptual system for face recognition is not a binary system; rather the system changes gradually in the amount of involvement of right-hemispheric processing with increasing exposure (Dahl et al., 2013). In the case of the chimpanzee participants that lived in captivity for most of their lives, we observe a systematic adaptation of righthemispheric processing from one class of faces, the chimpanzee faces, which the perceptual system tuned to due to early life processes, such as perceptual narrowing (Pascalis et al., 2002; McKone and Crookes, 2007), to another class, here the human faces, toward which the system adapted to due to lifelong and constant exposure of novel exemplars, i.e., perceptual learning (Goldstone, 1998; Bukach et al., 2006). Along the same line, it has been shown that the right-hemispheric dominance in processing faces is not prespecified, but requires early visual experience (Le Grand et al., 2003) and that expertise for cars and birds elicit righthemispheric activation predominantly in human "face areas" (Gauthier et al., 2000), hence reflecting the degree to which the face perception system stays plastic and adapts to the environmental conditions.

Assuming the left hemisphere is involved in analytic processing and the right hemisphere in configural processing (Bradshaw and Nettleton, 1981), the perceptual systems of our participants, showing biases to the left-chimeric faces in chimpanzee and human faces, are configurally processing both types of faces to some extent. Hence, the gradual change in processing strategies seems to reflect a balanced interplay of configural and part-based processes weighted according to the tuning of the participant's visual system toward a face class. This can be explained by the fact that chimpanzee and human faces are to some degree similar and share facial features or configurations (Dahl et al., 2013). This view is entirely compatible with neural data suggesting overlapping regions or neurons responding to human and macaque faces in the macaque brain (Sato et al., 2009).

The presented findings are not only suggestive for a greater involvement of the right hemisphere of the brain in face perception, but also for a right-hemispheric dominance in expert-face processing relying on configural/holistic processing strategies. Given numerous findings of hemispherically unbiased face representations in the brains of macaques (Bell et al., 2011; Ku et al., 2011; Popivanov et al., 2012), we cannot generalize that cerebral asymmetries are a common heritage of primates. However, we present a further piece of behavioral evidence in favor of functional asymmetry as a key principle of primate cognition that might serve as the basis from which the human species has evolved mechanisms to account for more specific functions in vision, communication, and language as well as manual control (Gunturkun, 2012).

\section{References}

Bell AH, Malecek NJ, Morin EL, Hadj-Bouziane F, Tootell RB, Ungerleider LG (2011) Relationship between functional magnetic resonance imaging-identified regions and neuronal category selectivity. J Neurosci 31:12229-12240. CrossRef Medline

Bouvier SE, Engel SA (2006) Behavioral deficits and cortical damage loci in cerebral achromatopsia. Cereb Cortex 16:183-191. Medline

Bradshaw JL, Nettleton NC (1981) The nature of hemisphericspecialization in man. Behav Brain Sci 4:51-63. CrossRef

Broman M (1978) Reaction-time differences between the left and right hemispheres for face and letter discrimination in children and adults. Cortex 14:578-591. CrossRef Medline

Bukach CM, Gauthier I, Tarr MJ (2006) Beyond faces and modularity: the power of an expertise framework. Trends Cogn Sci 10:159-166. CrossRef Medline

Burt DM, Perrett DI (1997) Perceptual asymmetries in judgements of facial attractiveness, age, gender, speech and expression. Neuropsychologia 35: 685-693. CrossRef Medline

Butler S, Gilchrist ID, Burt DM, Perrett DI, Jones E, Harvey M (2005) Are the perceptual biases found in chimeric face processing reflected in eyemovement patterns? Neuropsychologia 43:52-59. CrossRef Medline

Campanella S, Joassin F, Rossion B, De Volder A, Bruyer R, Crommelinck M (2001) Association of the distinct visual representations of faces and names: a PET activation study. Neuroimage 14:873-882. CrossRef Medline

Chaudhuri A, Nissanov J, Larocque S, Rioux L (1997) Dual activity maps in primate visual cortex produced by different temporal patterns of zif268 mRNA and protein expression. Proc Natl Acad Sci U S A 94:2671-2675. CrossRef Medline

Dahl CD, Wallraven C, Bülthoff HH, Logothetis NK (2009) Humans and macaques employ similar face-processing strategies. Curr Biol 19:509_ 513. CrossRef Medline

Dahl CD, Logothetis NK, Bülthoff HH, Wallraven C (2010) The Thatcher illusion in humans and monkeys. Proc Biol Sci 277:2973-2981. CrossRef Medline

Dahl CD, Logothetis NK, Bülthoff HH, Wallraven C (2011) Second-order relational manipulations affect both humans and monkeys. PLoS One 6:e25793. CrossRef Medline

Dahl CD, Rasch MJ, Tomonaga M, Adachi I (2013) Developmental processes in face perception. Sci Rep 3:1044. CrossRef Medline

Ellis HD, Shepherd JW (1975) Recognition of upright and inverted faces presented in the left and right visual fields. Cortex 11:3-7. CrossRef Medline

Gauthier I, Tarr MJ, Anderson AW, Skudlarski P, Gore JC (1999) Activation of the middle fusiform 'face area' increases with expertise in recognizing novel objects. Nat Neurosci 2:568-573. CrossRef Medline

Gauthier I, Anderson AW, Skudlarski P, Gore JC (2000) Expertise for cars and birds recruits right hemisphere face areas. Nat Neurosci 3:191-197

Gazzaniga MS, Smylie CS (1983) Facial recognition and brain asymmetries-clues to underlying mechanisms. Ann Neurol 13:536-540. CrossRef Medline

Geffen G, Bradshaw JL, Wallace G (1971) Interhemispheric effects on reaction time to verbal and nonverbal visual stimuli. J Exp Psychol 87:415422. CrossRef Medline

Gilbert C, Bakan P (1973) Visual asymmetry in perception of faces. Neuropsychologia 11:355-362. CrossRef Medline

Golarai G, Ghahremani DG, Whitfield-Gabrieli S, Reiss A, Eberhardt JL, Gabrieli JD, Grill-Spector K (2007) Differential development of highlevel visual cortex correlates with category-specific recognition memory. Nat Neurosci 10:512-522. Medline

Goldstone RL (1998) Perceptual learning. Annu Rev Psychol 49:585-612. CrossRef Medline

Grill-Spector K, Knouf N, Kanwisher N (2004) The fusiform face area subserves face perception, not generic within-category identification. Nat Neurosci 7:555-562. CrossRef Medline

Gunturkun O (2012) Brain asymmetry in vertebrates. In: How animals see the world: comparative behavior, biology, and evolution of vision (Lazareva OF, Shimizu T, Wasserman EA, eds). Oxford Scholarship Online. CrossRef

Guo K, Meints K, Hall C, Hall S, Mills D (2009) Left gaze bias in humans, rhesus monkeys and domestic dogs. Anim Cogn 12:409-418. CrossRef Medline 
Hamilton CR (1977) An assessment of hemispheric specialization in monkeys. Ann N Y Acad Sci 299:222-232. CrossRef

Hamilton CR (1983) Lateralization for orientation in split-brain monkeys. Behav Brain Res 10:399-403. CrossRef Medline

Hamilton CR, Vermeire BA (1988) Complementary hemispheric specialization in monkeys. Science 242:1691-1694. CrossRef Medline

Hilliard RD (1973) Hemispheric laterality effects on a facial recognition task in normal subjects. Cortex 9:246-258. CrossRef Medline

Hsiao JH, Cottrell G (2008) Two fixations suffice in face recognition. Psychol Sci 19:998-1006. CrossRef Medline

Ishai A, Ungerleider LG, Haxby JV (2000) Distributed neural systems for the generation of visual images. Neuron 28:979-990. CrossRef Medline

Kanwisher N, McDermott J, Chun MM (1997) The fusiform face area: a module in human extrastriate cortex specialized for face perception. J Neurosci 17:4302-4311. Medline

Kelly DJ, Quinn PC, Slater AM, Lee K, Ge L, Pascalis O (2007) The otherrace effect develops during infancy: evidence of perceptual narrowing. Psychol Sci 18:1084-1089. CrossRef Medline

$\mathrm{Ku}$ SP, Tolias AS, Logothetis NK, Goense J (2011) fMRI of the faceprocessing network in the ventral temporal lobe of awake and anesthetized macaques. Neuron 70:352-362. CrossRef Medline

Le Grand R, Mondloch CJ, Maurer D, Brent HP (2003) Expert face processing requires visual input to the right hemisphere during infancy. Nat Neurosci [Erratum (2003) 6:1329] 6:1108-1112. Medline

Levy J, Trevarthen C, Sperry RW (1972) Reception of bilateral chimeric figures following hemispheric deconnexion. Brain 95:61-78. CrossRef Medline

McCarthy G, Puce A, Gore JC, Allison T (1997) Face-specific processing in the human fusiform gyrus. J Cogn Neurosci 9:605-610. CrossRef

McKone E, Crookes K (2007) Understanding the developmental origins of primate face recognition: theoretical commentary on Martin-Malivel and Okada (2007). Behav Neurosci 121:1437-1441. CrossRef Medline

Mertens I, Siegmund H, Grüsser OJ (1993) Gaze motor asymmetries in the perception of faces during a memory task. Neuropsychologia 31:989-998. CrossRef Medline

Michel C, Rossion B, Han J, Chung CS, Caldara R (2006) Holistic processing is finely tuned for faces of one's own race. Psychol Sci 17:608-615. CrossRef Medline

Miller MB, Kingstone A, Gazzaniga MS (2002) Hemispheric encoding asymmetry is more apparent than real. J Cogn Neurosci 14:702-708. CrossRef Medline

Morris JP, Pelphrey KA, McCarthy G (2007) Face processing without awareness in the right fusiform gyrus. Neuropsychologia 45:3087-3091. CrossRef Medline

Morris RD, Hopkins WD (1993) Perception of human chimeric faces by chimpanzees - evidence for a right-hemisphere advantage. Brain Cogn 21:111-122. CrossRef Medline

Overman WH Jr, Doty RW (1982) Hemispheric specialization displayed by man but not macaques for analysis of faces. Neuropsychologia 20:113-128. CrossRef Medline

Parr LA, Hecht E, Barks SK, Preuss TM, Votaw JR (2009) Face processing in the chimpanzee brain. Curr Biol 19:50-53. CrossRef Medline

Pascalis O, de Haan M, Nelson CA (2002) Is face processing species-specific during the first year of life? Science 296:1321-1323. CrossRef Medline

Peirce JW, Leigh AE, Kendrick KM (2000) Configurational coding, familiarity and the right hemisphere advantage for face recognition in sheep. Neuropsychologia 38:475-483. CrossRef Medline

Perrett DI, Mistlin AJ, Chitty AJ, Smith PA, Potter DD, Broennimann R, Harries M (1988) Specialized face processing and hemispheric asymmetry in man and monkey: evidence from single unit and reaction time studies. Behav Brain Res 29:245-258. CrossRef Medline

Plotnik J, Nelson PA, de Waal FB (2003) Visual field information in the face perception of chimpanzees (Pan troglodytes). Ann N Y Acad Sci 1000:9498. Medline

Popivanov ID, Jastorff J, Vanduffel W, Vogels R (2012) Stimulus representations in body-selective regions of the macaque cortex assessed with event-related fMRI. Neuroimage 63:723-741. CrossRef Medline

Rizzolatti G, Umiltà C, Berlucchi G (1971) Opposite superiorities of the right and left cerebral hemispheres in discriminative reaction time to physiognomical and alphabetical material. Brain 94:431-442. CrossRef Medline

Rotshtein P, Vuilleumier P, Winston J, Driver J, Dolan R (2007) Distinct and convergent visual processing of high and low spatial frequency information in faces. Cereb Cortex 17:2713-2724. CrossRef Medline

Sato T, Uchida G, Tanifuji M (2009) Cortical columnar organization is reconsidered in inferior temporal cortex. Cereb Cortex 19:1870-1888. CrossRef Medline

Sergent J, Signoret JL (1992) Varieties of functional deficits in prosopagnosia. Cereb Cortex 2:375-388. CrossRef Medline

Sugita Y (2008) Face perception in monkeys reared with no exposure to faces. Proc Natl Acad Sci U S A 105:394-398. CrossRef Medline

Tsao DY, Freiwald WA, Knutsen TA, Mandeville JB, Tootell RB (2003) Faces and objects in macaque cerebral cortex. Nat Neurosci 6:989-995. CrossRef Medline

Tsao DY, Moeller S, Freiwald WA (2008) Comparing face patch systems in macaques and humans. Proc Natl Acad Sci U S A 105:19514-19519. CrossRef Medline

Vermeire BA, Hamilton CR (1998) Inversion effect for faces in split-brain monkeys. Neuropsychologia 36:1003-1014. CrossRef Medline

Vermeire BA, Hamilton CR, Erdmann AL (1998) Right-hemispheric superiority in split-brain monkeys for learning and remembering facial discriminations. Behav Neurosci 112:1048-1061. CrossRef Medline

Zangenehpour S, Chaudhuri A (2005) Patchy organization and asymmetric distribution of the neural correlates of face processing in monkey inferotemporal cortex. Curr Biol 15:993-1005. CrossRef Medline 